\title{
Alimentos inovadores: Comportamentos neofóbicos e desafios para as indústrias do setor
}

\section{Innovative food: Neophobic behavior and challenges for the sector industries}

\author{
Gislayne da Silva Goulart ${ }^{1}$ \\ Thelma Lucchese-Cheung ${ }^{1}$
}

\begin{abstract}
Resumo: Este artigo apresenta os resultados de um estudo exploratório que pretendeu analisar a atitude de 476 consumidores do município de Campo Grande quanto à sua percepção, motivação e tendência à neofobia alimentar quanto ao consumo de um produto alimentar inovador, o pão de queijo com cenoura desenvolvido pelo SENAI/MS. Para o tratamento dos dados, foi realizada a análise estatística de correspondência múltipla e análise de cluster com o auxílio do software francês SPAD. Os resultados dessas análises permitiram a composição de uma tipologia de comportamentos. Quatro grupos mais representativos daquela amostra foram definidos como: "Pão de queijo, só se for caseiro", "Adoro inovação, desde que a aparência do produto seja semelhante à do caseiro", "Confio mesmo em marca conhecida" e, por fim, "Nada disso me interessa". Os resultados mostraram que três dos quatro grupos de respondentes apresentaram tendências de rejeição ao produto alimentar inovador/não familiar. Comportamentos menos neofóbicos foram percebidos nos grupos melhor representados por mulheres, com as maiores rendas e níveis de informação. A falta de tempo ou habilidade no preparo do alimento e a habilidade da indústria em oferecer produtos parecidos com o caseiro ou com aquele que foi feito pela mãe/avó são as principais justificativas para as atitudes positivas em relação ao novo produto. Os resultados deste artigo representam para a indústria e outros agentes dos poderes público e privado importantes fontes de informação para construção de suas políticas.
\end{abstract}

Palavras-chave: Neofobia alimentar. Não familiar. Medo do novo.

\begin{abstract}
This article presents the results of an exploratory study that sought to analyze the attitude of 476 consumers in the city of Campo Grande about to their perception, motivation and tendency to food neophobia regarding the consumption of an innovative food product, the cheesy bread with carrot developed by SENAI/MS. Data were statistically treated by multiple correspondence analysis and cluster analysis with the help of the French software program SPAD. The results of these analyses allowed the composition of a typology of behaviors. The four most representative groups from that sample were defined as: "Cheese bread, only if it is homemade", "I like innovation, as long as the product appearance is similar to the homemade one", "I really trust in a well-known brand", and "None of that interests me". The results showed that three of the four groups of respondents tended to reject innovative/unfamiliar food products. Less neophobic behaviors were perceived in groups more represented by women with higher income and level of information. The lack of time or skill in the preparation of food and the ability of the sector to offer products similar to the homemade ones or to those made by mothers/grandmothers are the main reasons for the positive attitudes toward the new product. The results of this paper represent, for the industry and other public and private powers, important information sources for building their policies.
\end{abstract}

Keywords: Food neophobia, No-familiar; Fear of the new.

\section{Introdução}

O comportamento do consumidor pode ser considerado como uma temática que permite compreender a vida habitual dos indivíduos, seu cotidiano e o modo como interagem com produtos, serviços, o ambiente e com outros indivíduos (SOLOMON, 2006).

Estudos recentes conduzidos por Ventura (2010), bem como por empresas privadas interessadas em obter

informações acerca dos comportamentos de consumo dos indivíduos, por exemplo, o trabalho publicado pela Nielsen (2013) e pela Brasil Food Triends (FEDERAÇÃO..., 2010), revelaram que consumidores estão bastante interessados em adquirir novos produtos que lhes proporcionem praticidade, sobretudo no setor alimentício. Os resultados da Pesquisa Global da Nielsen sobre o Sentimento em Relação à Compra

\footnotetext{
${ }^{1}$ Universidade Federal de Mato Grosso do Sul - UFMS, CEP 79009-800, Campo Grande, MS, Brasil,

e-mail: gislaynegoulartgsg@gmail.com; thelma.lucchese@gmail.com
}

Recebido em 17/2/2014 — Aceito em 14/7/2014

Suporte financeiro: $\mathrm{CNPq}$. 
de Novos Produtos, a qual foi realizada em cinquenta e oito países por meio de entrevista virtual, destacam os alimentos e as bebidas como aqueles produtos novos que as pessoas declararam ter maior interesse em conhecer (NIELSEN, 2013).

No Brasil, o consumidor de alimentos demonstra uma mudança progressiva de comportamento. Mais informados e mais conscientes de seu papel no cuidado com a saúde, consumidores brasileiros demandam produtos que corroborem com esses valores (NIELSEN, 2012). Conforme os resultados do estudo ora mencionado, a Brasil Food Trends (FEDERAÇÃO..., 2010) revelou a existência de quatro grupos de tendência de consumo alimentar: Conveniência e Praticidade (34\%); Qualidade e Confiabilidade (23\%); Sensorialidade e Prazer (23\%); Saudabilidade/Bem-estar e Sustentabilidade/Ética (21\%).

Contudo, no campo da alimentação, apesar dessa tendência em adquirir produtos inovadores, de modo geral em todas as culturas, existem barreiras que podem influenciar o comensal a não consumir novos produtos. Para serem consumidos, os alimentos devem transpassar a barreira do corpo, e os aspectos simbólicos, próprios ao ato alimentar, são importantes determinantes das atitudes e dos comportamentos dos indivíduos (POULAIN, 2004). De acordo com Fischler (1995), o homem é um ser onívoro que se alimenta de carne, de vegetais e de símbolos. Além disso, explica que, enquanto onívoro, vive um constante paradoxo, porque a necessidade de variar sua alimentação para sobreviver lhe provoca ansiedade e medo do alimento novo, desconhecido e potencialmente perigoso (neofobia alimentar). Segundo o autor, as inovações no setor de alimentos são mais bem aceitas quando os indivíduos reconhecem algo de familiar no novo produto. Além disso, quanto à definição de neofobia alimentar, cabe acrescentar que esta pode ser considerada como o medo ou receio do novo, podendo variar de cultura para cultura e apresentar diferentes níveis (PLINER; HOBDEN, 1992).

Considerando-se os resultados dos estudos que revelam que os consumidores buscam por propostas inovadoras no setor de alimentos, bem como se levando em consideração o paradoxo do onívoro e a questão da neofobia alimentar, apresentados por Fischler (1995), interessou-se, neste presente artigo, em apresentar os resultados de um estudo exploratório que pretendeu analisar a atitude de quatrocentos e setenta e seis consumidores da cidade de Campo Grande, MS, quanto à sua percepção e motivação em consumir um produto alimentar inovador que foi desenvolvido pelo SENAI/MS, o pão de queijo com cenoura.

A relevância do estudo poderia ser justificada pelo número reduzido de trabalhos sobre a temática na literatura brasileira. Muitos trabalhos foram encontrados na literatura estrangeira. Contudo, no Brasil, os poucos trabalhos publicados focam, sobretudo, o comportamento de crianças em relação a um produto alimentar não familiar. O artigo está estruturado em uma seção introdutória; outra de revisão da literatura; uma sobre a metodologia empregada para coletar e analisar os dados; uma penúltima que trata da análise dos resultados e discussões; e, por fim, uma de conclusão.

\section{Revisão da literatura}

A análise da literatura disponível sobre o escopo deste trabalho obedeceu à necessidade de se buscar fundamentos sobre as questões que envolvem o comportamento do consumidor de alimentos perante um produto alimentar não familiar.

Compreender o comportamento do consumidor é extremamente relevante para que as empresas possam se desenvolver e se sustentar em um mercado muito competitivo (BLACKWELL; MINIARD; ENGEL, 2005). Os comportamentos dos consumidores devem ser investigados de maneira abrangente, em seus aspectos mais gerais, uma vez que são múltiplos os fatores que influenciam suas decisões de compra e consumo. Os autores sugerem três grupos de fatores que influenciam a tomada de decisão do consumidor, sendo eles: a) Diferenças individuais - recursos do consumidor, conhecimento, atitudes, motivação, personalidade, valores e estilo de vida; b) Influências ambientais - cultura, classe social, influência pessoal, família e situação; e c) Processos psicológicos - informação em processamento, aprendizagem e mudança de atitude e comportamento. Solomon (2006), entretanto, ressalta que, em estudos sobre comportamentos, maior atenção à variável cultura deve ser dada.

No que se refere ao estudo do comportamento do consumidor de alimentos, Steenkamp (1993) apresenta uma taxonomia sobre os determinantes do comportamento dividida em três grupos de fatores: os fatores relacionados ao próprio consumidor (biológico, psicológico e personalidade); o fator ambiente (sociocultural, econômico e marketing); e as propriedades dos alimentos (propriedades físicas, propriedades químicas e propriedades nutricionais). Segundo estudiosos, o fator ambiente tem uma influência significante, pois, em cada lugar, os comensais têm uma percepção diferente dos alimentos que podem ou não ultrapassar a barreira do seu corpo. Tais percepções estão ligadas às normas, regras e tabus que lhes são transmitidos pelos valores socioculturais e pelas posições sociais em que se encontram (POULAIN; PROENÇA, 2003; POULAIN, 2004; JOMORI; PROENÇA; CALVO, 2008).

Jomori, Proença e Calvo (2008) relatam que Furst et al. (1996) criaram um modelo teórico que ilustra o processo da escolha alimentar que 
pretende tornar mais dinâmica a forma de analisar o comportamento alimentar. Esse modelo apresenta três grandes componentes que corroboram para o entendimento do comportamento do comensal, sendo eles: o curso de vida - seu papel pessoal e o seu meio ambiente sociocultural; as influências - ideais, fatores individuais, recursos disponíveis, estrutura social e contexto alimentar; e o sistema pessoal - englobando o processo de negociação de valores e uma série de estratégias definidas para fazer a escolha alimentar. O estudo de Furst et al. (1996 apud JOMORI; PROENÇA; CALVO, 2008) bem como outros estudos apresentam indícios de que, em geral, todo o consumo sofre influência do efeito social, considerando o ambiente em que o consumidor está inserido (STEENKAMP, 1993; SCHIFFMAN; KANUNK, 2000; SHETH; MITTAL; NEWMAN, 2001; POULAIN; PROENÇA, 2003; POULAIN, 2004; SOLOMON, 2006). No processo de escolha do alimento, o ambiente em que o consumidor está inserido, suas experiências passadas, os meios dos quais esse consumidor participa e os significados simbólicos que esse produto pode ter para ele influenciam o seu comportamento de compra e consumo de um alimento inovador e não familiar.

Quando se trata do consumo de um produto alimentar não familiar, Ritchey et al. (2003) constataram que alguns indivíduos apresentam prazer em consumi-los e outros apresentam uma forte relutância. Conforme já mencionado, esse segundo comportamento, denominado neofobia alimentar, atua no subconsciente como uma função protetora em um ambiente de possíveis produtos nocivos ao organismo humano (PLINER; HOBDEN, 1992; YAMAMOTO; LOPES, 2004).

Segundo Visalberghi e Fragaszy (1995), a neofobia possui graus de rejeição que podem variar desde a completa rejeição em comer um novo alimento ou até a concordância em, somente, ingerir pequenas porções.

A maior parte dos estudos encontrados na literatura se interessara em investigar os fatores que possivelmente podem influenciar positivamente ou negativamente a ocorrência de neofobia em seres humanos. Tais investigações, em geral, preocuparam-se mais em saber a diferença de comportamento em relação ao alimento novo quanto ao gênero, à idade, à renda, à escolaridade, aos ambientes participados, à cultura e aos aspectos sensoriais do produto. Contudo, este trabalho constatou que os estudos a respeito das diferenças de gêneros quanto à neofobia alimentar apresentam resultados controversos. Alguns estudos sugerem que as mulheres tendem a ser mais neofóbicas (FRANK; KLAAUW, 1994; MOONEY; WALBOURN, 2001; NORDIN et al., 2004; YAMAMOTO; LOPES, 2006; SANJUÁN-LÓPEZ; PHILIPPIDIS; RESANO-EZCARAY, 2011); outros apontam os homens (KOIVISTO; SJÖDEN, 1996;
HURSTI; SJÖDÉN, 1997; TUORILA et al., 2001; BÄCKSTRÖM; PIRTTILÄ-BACKMAN; TUORILA, 2003; CAMARENA; SANJUÁN; PHILIPPIDIS, 2011); e há os que não encontraram diferenças significativas entre gêneros (PLINER; HOBDEN, 1992; MEISELMAN; KING; GILLETTE, 2010; D'ANTUONO; BIGNAMI, 2012; FERNÁNDEZRUIZ; CLARET; CHAYA, 2013).

Em relação à variável idade, há um maior consenso entre os estudos realizados com diferentes amostras (Quadro 1). Observou-se um maior nível de neofobia alimentar na infância que tende a se estabilizar até o início da fase adulta, mas que volta a aumentar novamente com a velhice (HURSTI; SJÖDÉN, 1997; BIRCH, 1999; DOVEY et al., 2008).

$\mathrm{O}$ fator socioeconômico tende a influenciar de maneira diferente o grau de neofobia alimentar. A renda, que influencia a composição da dieta do indivíduo, pode aumentar ou diminuir a probabilidade do indivíduo ter acesso a produtos alimentares em geral (SANJUÁN-LÓPEZ; PHILIPPIDIS; RESANOEZCARAY, 2011). A renda tende a estar relacionada positivamente com a escolaridade e, por isso, em estudos com pessoas com maiores rendas e maior nível de formação, maiores contatos com alimentos inovadores ocorrem quando comparados aos contatos com os mesmos alimentos entre grupos menos abastados e com menor escolaridade (MEISELMAN; KING; GILLETTE, 2010).

Além disso, pensando sobre os tipos de ambientes em que os indivíduos estão inseridos, a probabilidade de o indivíduo ter contato com produtos alimentares não familiares pode estar relacionada com o local em que habita e que frequenta. Em um estudo realizado por Tuorila et al. (2001), com uma amostra representativa da população finlandesa, encontrou-se uma diminuição da neofobia alimentar com o aumento do grau de urbanização. Tal constatação também foi observada em estudo com grupos de adolescentes australianos. Os adolescentes que viviam na zona urbana apresentavam nível de neofobia mais baixo quando comparado ao dos que viviam na zona rural (FLIGHT; LEPPARD; COX, 2003). Em ambos os estudos citados, o fator cultura está relacionado, direta ou indiretamente, pois se deve considerar que nos grandes centros as misturas culturais ampliam o campo de variedade e informação de produtos alimentares (FLIGHT; LEPPARD; COX, 2003).

Devido à variedade de fatores que podem levar à ocorrência de um comportamento de consumo mais neofóbico, julgou-se interessante apresentar o Quadro 1, o qual resume os fatores mais relatados na literatura que influenciam o nível de receio e/ou medo em adultos de consumir um produto alimentar não familiar. Torna-se relevante destacar que uma das dificuldades na comparação dos estudos é a não padronização das faixas etárias. Assim, para facilitar a 
Quadro 1. Fatores que influenciam o nível de receio e/ou medo em adultos.

\begin{tabular}{|c|c|c|c|c|c|}
\hline \multirow{2}{*}{ Autores } & \multirow{2}{*}{ Amostra } & \multicolumn{4}{|c|}{ Fatores } \\
\hline & & Gênero & Idade & Escolaridade & Renda \\
\hline Pliner e Hobden (1992) & 41,35 e 80 & ns & & & \\
\hline Frank e Klaauw (1994) & 719 & $f+$ & & & \\
\hline Koivisto e Sjöden (1996) & $231^{\mathrm{a} / \mathrm{c}}$ & $\mathrm{m}+$ & & & \\
\hline Hursti e Sjödén (1997) & $722^{\mathrm{c} *}$ & $\mathrm{~m}+$ & & & \\
\hline Mooney e Walbourn (2001) & 113 & $\mathrm{f}+$ & & & \\
\hline Tuorila et al. (2001) & 1083 & $\mathrm{~m}+$ & $\mathrm{b}+\mathrm{c}++$ & $\begin{array}{c}\text { Baixa+ } \\
\text { Méd+/- } \\
\text { Alta- }\end{array}$ & \\
\hline Bäckström, Pirttilä-Backman e Tuorila (2003) & $44^{g}$ & $\mathrm{~m}+$ & $\mathrm{b} / \mathrm{c}+$ & $\begin{array}{c}\text { Baixa+ } \\
\text { Alta- }\end{array}$ & \\
\hline Nordin et al. (2004) & 200 & $\mathrm{f}+$ & & & \\
\hline Schickenberg et al. (2006) & 326 & & & $\begin{array}{c}\text { Baixa+ } \\
\text { Alta- }\end{array}$ & \\
\hline Yamamoto e Lopes (2006) & 406 & $\mathrm{f}+$ & ns & ns & \\
\hline Meiselman, King e Gillette (2010) & 1567 e 6843 & ns** & $\mathrm{b} / \mathrm{c}+$ & $\begin{array}{c}\text { Baixa+ } \\
\text { Média+/- } \\
\text { Alta- }\end{array}$ & $\begin{array}{c}\text { Baixa+ } \\
\text { Média+/- } \\
\text { Alta- }\end{array}$ \\
\hline Camarena, Sanjuán e Philippidis (2011) & 270 & $\mathrm{~m}+$ & $\mathrm{c}+$ & & Alta+ \\
\hline Sanjuán-López, Philippidis e Resano-Ezcaray (2011) & 208 & $\mathrm{f}+$ & $\mathrm{c}+$ & Baixa+ & Baixa+ \\
\hline D’Antuono e Bignami (2012) & 283 & ns & $\mathrm{c}+$ & Baixa+ & \\
\hline Fernández-Ruiz, Claret e Chaya (2013) & 309 & ns & $\begin{array}{l}\mathrm{a}- \\
\mathrm{c}+\end{array}$ & & \\
\hline
\end{tabular}

a/c = adultos e crianças; $\mathrm{c}^{*}=$ crianças de 7 a 17 anos (+familiares não contabilizados, para o presente estudo será retratado somente o comportamento dos pais); $\mathrm{g}=$ grupo focal $(\mathrm{n}=9)$; $\mathrm{ns}=$ não significante; $* *=$ Pouco ou nenhum efeito do gênero sobre a neofobia; $f=$ feminino; $m=$ masculino; $a=$ menos de 30 anos; $b=30$ a 50 anos; $c=50$ anos ou mais; $(+)=$ mais neofobia; $(-)=$ menos neofobia. Fonte: Elaborado pelas autoras.

apresentação dos resultados desses estudos, optou-se por agrupar os resultados em: A [indivíduos com menos de 30 anos]; B [de 30 a 50 anos]; e C [de 50 anos ou mais].

Os dados apresentados no Quadro 1 serão utilizados por este artigo no capítulo de apresentação e discussão dos resultados. Pretende-se utilizar os resultados daquelas pesquisas para melhor compreender os comportamentos neofóbicos declarados pelos consumidores do estudo empírico, bem como identificar os principais fatores que poderiam justificar tais comportamentos.

\section{METODOLOGIA}

O presente estudo é de caráter exploratóriodescritivo, esse método incide na descrição de determinada população ou fenômeno, expondo características e aspectos (VERGARA, 2005).

Para o cálculo do tamanho da amostra, considerou-se uma população infinita. Utilizando um intervalo de confiança de $95 \%$ e erro padrão de $5 \%$, com base na fórmula (1) para o cálculo do tamanho da amostra aleatória simples para descrição da proporção populacional (BUSSAB; MORETTIN, 2011). Os cálculos indicaram a necessidade de coletar dados de, no mínimo, 385 indivíduos. Contudo, para maior segurança, foram coletados os dados de 490 consumidores de alimentos, o que, consequentemente, fez o erro padrão chegar a $4,4 \%$, como pode ser observado na Equação 2.

$$
\begin{aligned}
n & =\left(\frac{z}{e}\right)^{2} \cdot p \cdot(1-p) \\
n & =\left(\frac{1,96}{0,05}\right)^{2} \cdot 0,25 \quad n=384,16 \\
400 & =\left(\frac{1,96}{e}\right)^{2} \cdot 0,25 \quad e=0,044
\end{aligned}
$$

No que concerne à coleta de dados, utilizou-se o método survey, com base em um corte transversal, com variáveis quantitativas e qualitativas (MALHOTRA, 2011). Contou-se com o auxílio de um questionário estruturado, composto por questões abertas e fechadas (CRESWELL, 2007; MATTAR, 2007; MALHOTRA, 2011), tendo sido aplicado na amostra definida. Cabe, todavia, ressaltar, que, devido à existência de missingvaluese outliers, 14 questionários foram descartados, restando 476 questionários válidos. No questionário, foram coletados dados sobre o perfil socioeconômico dos indivíduos e sobre as 
suas maneiras de perceber um produto alimentar familiar [pão de queijo/pão de queijo congelado] e um produto alimentar não familiar [pão de queijo com cenoura criado pelo SENAI/MS]. Contudo, como este artigo está inserido em um estudo maior, financiado pelo CNPq e, considerando seu objetivo principal, optou-se por utilizar somente as questões fechadas contidas no questionário.

Cabe ressaltar que, para conhecer como os consumidores tendem a se comportar quando são levados a pensar sobre um produto alimentar inovador, foi questionado, em uma escala de importância [(1) o que eu iria pensar em primeiro lugar; (2) o que eu iria pensar em segundo lugar; (3) o que eu iria pensar em terceiro lugar; (4) a última coisa em que eu iria pensar], se fosse lançado um novo sabor, por exemplo, pão de queijo com cenoura, em que ordem você pensaria sobre as seguintes alternativas: "Isso não é pão de queijo de verdade"; "Quais os ingredientes que eles mudaram nessa receita?"; "Isso é comida de criança ou de idosos"; "Duvido que seja bom"; "Pode ser interessante, porque é mais saudável"; e "Será que é um produto menos calórico?". E, considerando uma escala de concordância de 4 pontos, questionou-se, também, qual o grau de concordância do consumidor para a afirmativa "será difícil fazer o consumidor escolher uma embalagem de um produto como esse". Assim, motivados pelo referido questionamento, interessou-se pelas respostas que melhor representaram cada grupo encontrado do quadrante fatorial analisado.

Os dados foram tratados, utilizando o software francês SPAD. Como técnicas estatísticas de tratamento de dados, optou-se pela realização de métodos de descrição estatística elementar, análise de correspondência múltipla (ACM) e a análise de cluster. Ao realizar a análise descritiva dos dados, foi possível identificar o perfil da amostra estudada, Tabela 1.

A adoção da análise de correspondência múltipla (ACM) se justifica devido à técnica permitir avaliar um grupo grande de variáveis. Essa técnica calcula a distância entre variáveis ativas, o que possibilita observar indivíduos e modalidades de variáveis que se aproximam ao serem representadas em um

Tabela 1. Perfil da amostra.

\begin{tabular}{|c|c|c|c|}
\hline \multicolumn{3}{|c|}{ Homens } & \multirow[b]{2}{*}{ Situação Conjugal } \\
\hline Faixa Etária & Escolaridade & Renda do domicílio & \\
\hline Até 20 anos & Analfabeto & até 1 salário mínimo & Solteiro \\
\hline $15,61 \%$ & $0,98 \%$ & $10,24 \%$ & $51,71 \%$ \\
\hline 20 a 30 anos & E. Fundamental & até $\mathrm{R} \$ 2034,00$ & Casado \\
\hline $40,49 \%$ & $6,83 \%$ & $33,17 \%$ & $38,54 \%$ \\
\hline 30 a 40 anos & Ensino Médio & até $\mathrm{R} \$ 3390,00$ & Viúvo \\
\hline $24,39 \%$ & $40 \%$ & $21,95 \%$ & $0,98 \%$ \\
\hline 40 a 50 anos & Ensino superior & até $\mathrm{R} \$ 10170,00$ & Mora junto \\
\hline $8,78 \%$ & $39,51 \%$ & $26,34 \%$ & $8,78 \%$ \\
\hline 50 a 60 anos & Pós-graduação & mais de $\mathrm{R} \$ 10170,00$ & \\
\hline $8,78 \%$ & $12,68 \%$ & $8,29 \%$ & \\
\hline \multicolumn{4}{|l|}{ mais de 60 anos } \\
\hline \multicolumn{4}{|l|}{$1,95 \%$} \\
\hline \multicolumn{3}{|c|}{ Mulheres } & $n=271$ \\
\hline Faixa Etária & Escolaridade & Renda do domicílio & Situação Conjugal \\
\hline Até 20 anos & Analfabeto & até 1 salário mínimo & Solteira \\
\hline $15,50 \%$ & $1,48 \%$ & $14,02 \%$ & $52,40 \%$ \\
\hline 20 a 30 anos & E. Fundamental & até $\mathrm{R} \$ 2034,00$ & Casada \\
\hline $33,58 \%$ & $4,43 \%$ & $26,57 \%$ & $36,53 \%$ \\
\hline 30 a 40 anos & Ensino Médio & até $\mathrm{R} \$ 3390,00$ & Viúva \\
\hline $29,15 \%$ & $38,01 \%$ & $24,72 \%$ & $3,69 \%$ \\
\hline 40 a 50 anos & Ensino superior & até $\mathrm{R} \$ 10170,00$ & Mora junto \\
\hline $11,44 \%$ & $41,70 \%$ & $27,68 \%$ & $7,38 \%$ \\
\hline 50 a 60 anos & Pós-graduação & mais de $\mathrm{R} \$ 10170,00$ & \\
\hline $8,12 \%$ & $14,39 \%$ & $7,01 \%$ & \\
\hline \multicolumn{4}{|l|}{ mais de 60 anos } \\
\hline $2,21 \%$ & & & \\
\hline
\end{tabular}

Fonte: Elaborado pelas autoras. 
espaço multidimensional (LUCCHESE-CHEUNG; BATALHA; LAMBERT, 2012). Essa técnica produz representações gráficas, sendo que os objetos a serem descritos aparecem como pontos sobre um plano ou uma reta (MINGOTI, 2005).

Já o agrupamento, ou análise de cluster, possibilita dividir os elementos da amostra em grupos de maneira que

[...] os elementos pertencentes a um mesmo grupo sejam similares entre si com respeito às variáveis (características) que neles foram medidas, e os elementos em grupos diferentes sejam heterogêneos em relação a estas mesmas características [...] (MINGOTI, 2005, p. 155).

Essas duas técnicas são complementares, permitindo o uso simultâneo de ambas. Segundo LuccheseCheung, Batalha e Lambert (2012, p. 119), “[...] a distância de um grupo do centro do plano fatorial corresponde a sua diferença em relação aos indivíduos situados no ponto central". Os autores complementam que, quanto mais afastados os grupos estiverem do ponto central, maior será o coeficiente de correlação entre as variáveis do cluster e o eixo fatorial.

Destaca-se que as ligações entre as modalidades das variáveis são calculadas pelo teste do quiquadrado de Person. Considerando que os valores superiores a 2 em valor absoluto são correspondentes a um nível de significância de 5\%, isto é, são utilizadas as modalidades com maior relação entre si para definir uma classe. Assim, a partir da análise das semelhanças entre duas modalidades diferentes, que serão consideradas mais próximas quando forem mais escolhidas ou rejeitadas por um número significativo de respondentes, determinam as características de cada classe (LEBART et al., 1995; ESCOFIER; PAGES, 1998 apud LUCCHESE-CHEUNG, 2007).

É importante pontuar que o número de grupos de indivíduos foi pré-determinado em função do grau de síntese desejado e que as variáveis ativas utilizadas neste estudo, para a análise de correspondência múltipla, foram as questões fechadas referentes ao consumo de pão de queijo tradicional congelado e a percepção de um produto alimentar não familiar [pão de queijo com cenoura]. E, para testar o que determinam as diferentes percepções declaradas, foram utilizadas as variáveis ilustrativas, ou seja, aquelas que representam as características socioeconômicas e culturais dos indivíduos como determinantes dos seus comportamentos. A análise de correspondência múltipla permitiu recuperar os principais traços de comportamentos e seus determinantes. Já a análise de cluster nos possibilitou formar os grupos de comportamentos semelhantes.

\section{ANÁLISE DOS RESULTADOS E DISCUSSÃO}

Pretendendo-se obter informações sobre os comportamentos de consumo em relação a um alimento inovador que será colocado no mercado nos próximos meses, buscou-se com as técnicas estatísticas de tratamento dos dados coletados em campo (ACM e cluster) agrupar os indivíduos em função da similaridade das suas respostas. Com isso, foi possível analisar quais as motivações em consumir ou não o produto inovador e analisar a ocorrência de graus de neofobia alimentar na amostra da pesquisa. Os posicionamentos dos grupos nos quadrantes fatoriais podem ser verificados na Figura 1.

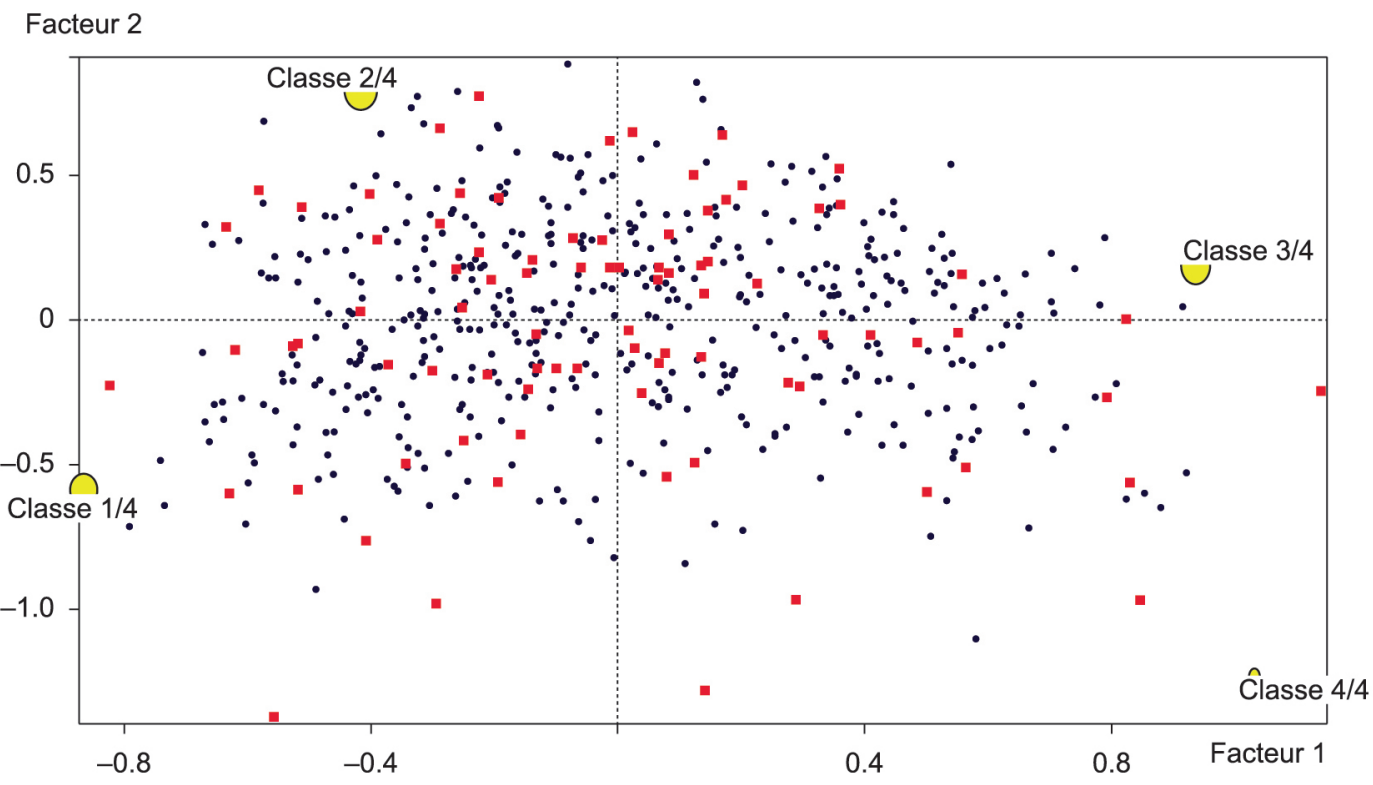

Figura 1. Localização de cada grupo no plano fatorial. Fonte: Elaborado pelas autoras. 
A análise das modalidades que melhor contribuíram para a formação dos eixos fatoriais permitiu que oposições marcantes quanto às maneiras como os grupos declararam pensar sobre o consumo de pão de queijo congelado fossem verificadas, Figura 2. Constatou-se que o grupo 2/4 - "Pão de queijo, só se for caseiro" - é o maior grupo em representatividade de respostas escolhidas de modo semelhante pela maior parte dos entrevistados. Já o grupo 4/4 - "Nada disso me interessa" - é o menor em representatividade das respostas. Ambos os grupos mencionados, "Pão de queijo, só se for caseiro" e "Nada disso me interessa", se encontram em quadrantes opostos e, considerando o objetivo deste artigo, concentrar-nos-emos nestas oposições. Cabe acrescentar que tais oposições devem ser compreendidas como diferenças motivacionais e comportamentais para o fato estudado. Tomando-se os dois grupos ora mencionados como exemplo, julga-se então interessante observar as modalidades de variáveis que melhor representam as opiniões sobre o consumo de pão de queijo tradicional e suas motivações e percepções de um produto inovador, o pão de queijo com cenoura.

O grupo 1/4 - "Adoro inovação, desde que a aparência do produto seja semelhante à do caseiro" -, representa $28,99 \%$ da amostra, é mais bem representado por indivíduos do gênero feminino, por pessoas casadas, com pós-graduação; por faixas etárias entre 30-40, 40-50 e 50-60 anos, com renda até $\mathrm{R} \$ 10.170,00$ e que compram pão de queijo congelado a cada 2 ou 3 meses. Ou seja, considera-se que tal perfil melhor descreve os indivíduos que consomem pão de queijo congelado com maior frequência. Os respondentes deste grupo declararam que são mais interessados em experimentar sabores e versões diferentes do pão de queijo tradicional e que a marca conhecida é vista em primeiro lugar ao observarem uma embalagem desse produto no ponto de venda. O grupo concorda que um bom pão de queijo faz lembrar o feito pela mãe/avó, "o caseiro", e que os pães de queijo congelados estão ficando tão bons que até parecem que foram feitos em casa. Os integrantes deste grupo discordam totalmente de que pão de queijo é um produto que serve para substituir uma refeição.

Todas essas descrições de comportamentos declarados de consumo, bem como o perfil sociodemográfico e econômico representativo do grupo, poderiam apontar uma tendência menos neofóbica desse grupo em relação ao pão de queijo com cenoura. Conforme constatado por outros estudos, quanto maior o nível de escolaridade e renda, menor o nível de neofobia (TUORILA et al., 2001; BÄCKSTRÖM; PIRTTILÄ-BACKMAN; TUORILA, 2003; SCHICKENBERG et al., 2006; MEISELMAN; KING; GILLETTE, 2010). Outro fato é que as mulheres representaram melhor esse grupo, o que

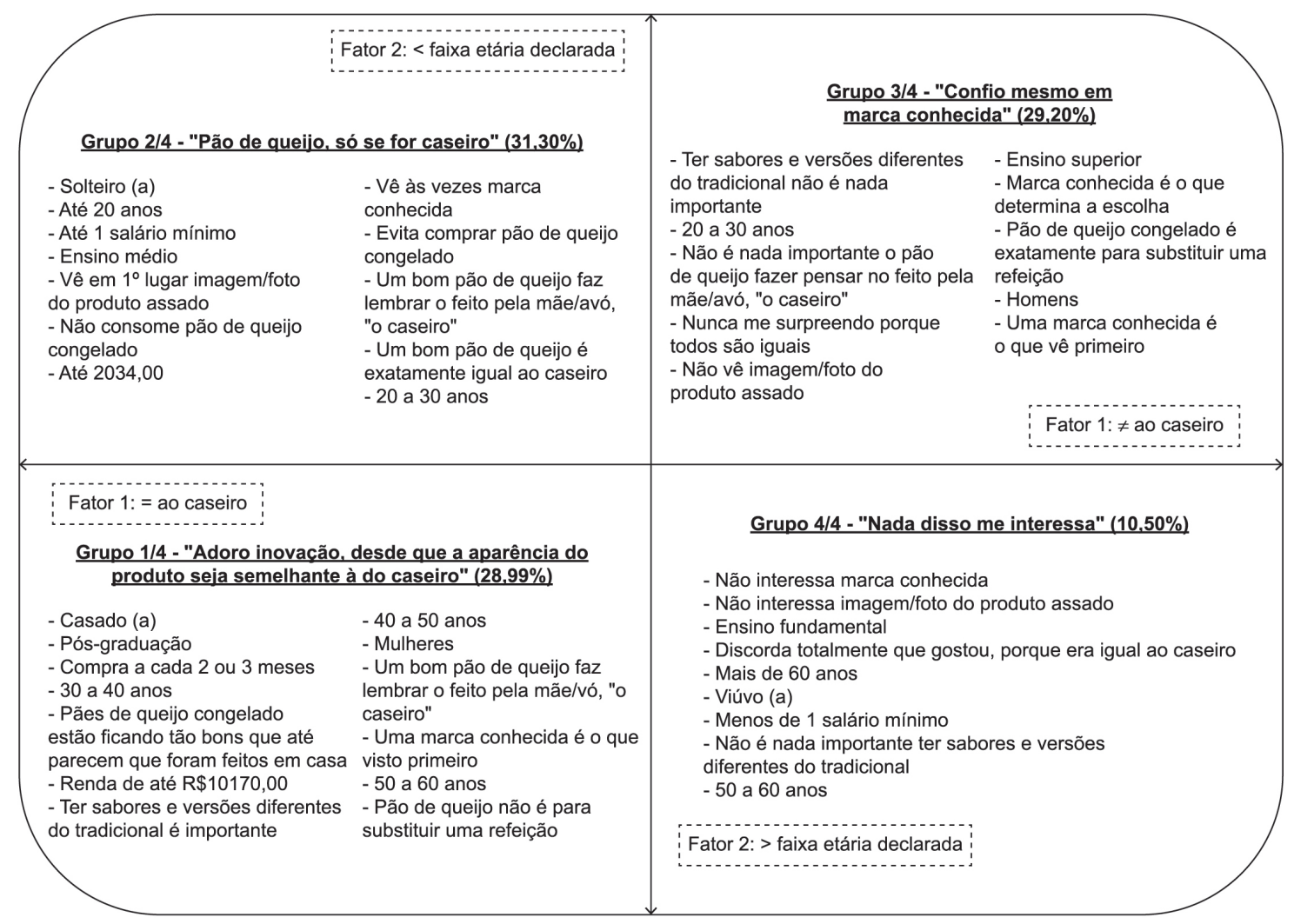

Figura 2. Modalidades de variáveis que melhor caracterizaram a resposta dos grupos. Fonte: Elaborado pelas autoras. 
corrobora com os estudos que apontam que as mulheres são menos neofóbicas (KOIVISTO; SJÖDEN, 1996; HURSTI; SJÖDÉN, 1997; TUORILA et al., 2001; BÄCKSTRÖM; PIRTTILÄ-BACKMAN; TUORILA, 2003; CAMARENA; SANJUÁN; PHILIPPIDIS, 2011). A afirmação de que consideram importante que as indústrias apresentem novos sabores e versões diferentes do pão de queijo tradicional também justifica uma atitude menos neofóbica.

Portanto, esse grupo apresenta forte tendência de aceitar provar o produto inovador, não familiar, mais facilmente. Essa tendência pode ser confirmada também, devido às declarações como quando questionado "se fosse lançado um novo sabor de pão de queijo, por exemplo: 'pão de queijo com cenoura', o que você pensaria em ordem de importância" e "qual é o seu grau de concordância para a afirmativa "será difícil fazer o consumidor escolher uma embalagem de um produto como esse" " [como descrito na metodologia]. As respostas que melhor caracterizam esse grupo conforme o grau de representatividade na análise de cluster podem ser verificas na Figura 3.

O grupo 3/4 - "Confio mesmo em marca conhecida", que está em quadrante oposto ao grupo "Adoro inovação, desde que a aparência do produto seja semelhante à do caseiro", é composto por $29,20 \%$ da amostra. Foram mais bem representados por homens, de 20 a 30 anos, e por pessoas que têm ensino superior. Os indivíduos do grupo declararam que a marca conhecida é o que veem primeiro na embalagem e, consequentemente, a marca conhecida é o que mais determinaria sua escolha no ponto de venda. O pão de queijo para esse grupo tende a ser um produto mais funcional do que hedônico, pois pão de queijo congelado é um ótimo substituto de uma refeição, quando não se tem nada para comer ou quando não se quer cozinhar. Os respondentes desse grupo também afirmaram que não é nada importante que a indústria se preocupe em lançar sabores e versões diferentes do tradicional e que faça com que fiquem parecidos com aqueles que foram feitos pela mãe/avó. Além disso, no ponto de venda, não olham para a imagem/foto do produto assado na embalagem. Esse grupo tende a ser fiel à marca, não procura semelhanças com o caseiro, mas também não quer que o produto seja diferente do que estão acostumados a consumir.

As respostas do grupo levam à constatação de uma tendência à neofobia alimentar maior do que a do grupo anteriormente analisado. Poder-se-ia considerar que, para a amostra estudada, homens, em relação às mulheres, são mais neofóbicos no que se refere à proposta de produto inovador. Além disso, tal comportamento poderia ser justificado por outros fatores determinantes do comportamento, como a cultura local (MOONEY; WALBOURN, 2001; POULAIN, 2004; FLANDRIN; MONTANARI, 2009). A tendência do grupo em rejeitar o produto inovador, não familiar, é confirmada pelas declarações dadas quando levados a pensar sobre tal, ver Figura 4.

A análise da oposição fatorial dos grupos "Adoro inovação, desde que a aparência do produto seja semelhante à do caseiro" e "Confio mesmo em marca conhecida" em função das modalidades de variáveis que representam melhor seus comportamentos em relação ao produto pão de queijo tradicional e o produto inovador, nos permite fazer algumas observações que podem ser interessantes para os agentes do setor privado de alimentos. A distinção dos gêneros em relação à opinião sobre o produto inovador foi evidenciada pelo interesse de mulheres pelo produto não familiar, pelas novas versões e novos sabores. Já os homens revelaram preferência pelo produto tradicional, que respeite o que é habitualmente conhecido por eles

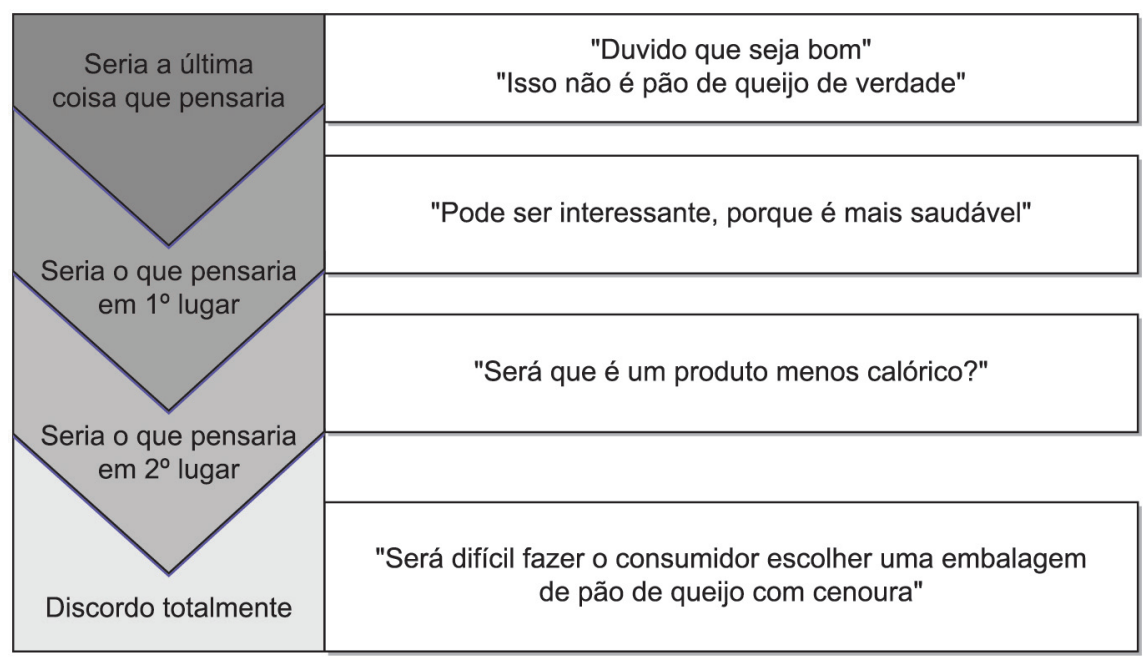

Figura 3. O que o grupo "Adoro inovação, desde que a aparência do produto seja semelhante à do caseiro" pensa sobre um pão de queijo com cenoura. Fonte: Elaborado pelas autoras. 


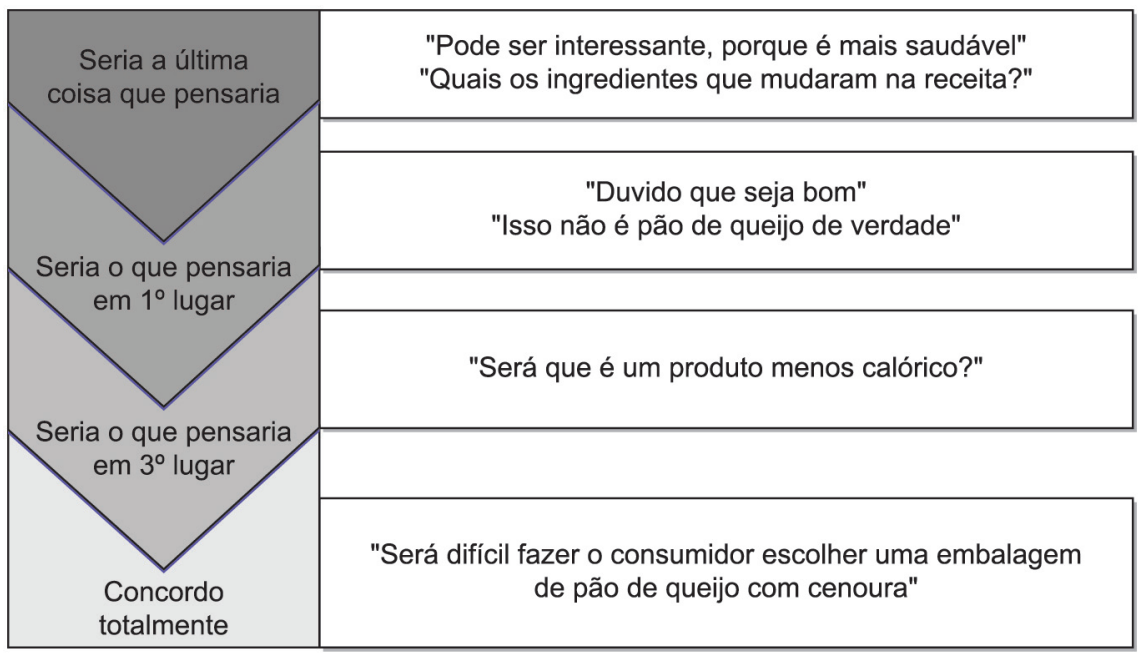

Figura 4. O que o grupo "Confio mesmo em marca conhecida" pensa sobre um pão de queijo com cenoura. Fonte: Elaborado pelas autoras.

como "pão de queijo genuinamente tradicional". Essa tendência corrobora com os estudos de Koivisto e Sjöden (1996), Hursti e Sjödén (1997), Tuorila et al. (2001), Bäckström, Pirttilä-Backman e Tuorila (2003) e Camarena, Sanjuán e Philippidis (2011) que revelam que os homens tendem a serem mais neofóbicos. Mooney e Walbourn (2001) expõem que um dos principais fatores considerados para a rejeição do alimento entre os homens é o sabor, pois esses tendem a rejeitar mais vegetais do que as mulheres. Outros estudos também constataram que os indivíduos do gênero feminino tendem a consumir mais legumes que os do gênero masculino por influências socioculturais e biológicas (MACAUX, 2001; ROBINSON; THOMAS, 2004; COOKE;WARDLE, 2005).

Em relação ao grupo 2/4 - "Pão de queijo, só se for caseiro", que representa $31,30 \%$ dos respondentes, este tem como fator caracterizante a situação conjugal [solteiro(a)], mais bem representado pelas faixas etárias até 20 anos e de 20 a 30 anos, com ensino médio e renda do domicílio compreendida em até 1 salário mínimo e/ou até R \$ 2034,00. Esse grupo é composto por consumidores jovens que declararam não consumir pão de queijo congelado, também afirmaram que evitam comprar, porque nunca acham que são bons como os feitos em casa e a creditam que um bom pão de queijo seria exatamente igual ao caseiro, fazendo-os pensar "como era bom o pão de queijo da minha mãe/avó!". Assim, se tivessem que comprar pão de queijo congelado, os indivíduos do grupo analisado observariam em primeiro lugar a imagem/foto na embalagem que representasse o produto assado e, às vezes, a marca conhecida. Ou seja, se tivessem que comprar um produto congelado, teriam de fazer um grande esforço para conseguir categorizá-lo no seu campo de representações, já que nem o produto tradicional lhes é familiar.

O grupo "Pão de queijo, só se for caseiro" apresenta uma tendência à neofobia alimentar em relação ao produto tradicional [pão de queijo congelado] e ao produto inovador, não familiar. Com base na literatura, esse fato poderia ser justificado, primeiramente, pela renda familiar baixa que tende a limitar a variedade de produtos alimentares na dieta, seguido do nível de escolaridade médio que tende a influenciar moderadamente essa pouca familiaridade com o produto (MOONEY; WALBOURN, 2001; TUORILA et al., 2001; BÄCKSTRÖM; PIRTTILÄBACKMAN; TUORILA, 2003; POULAIN, 2004; SCHICKENBERG et al., 2006; FLANDRIN; MONTANARI, 2009; MEISELMAN; KING; GILLETTE, 2010; SANJUÁN-LÓPEZ; PHILIPPIDIS; RESANO-EZCARAY, 2011).

Em relação ao produto inovador, o pão de queijo com cenoura, os respondentes tendem, principalmente, a questionar sobre sua composição, o que comprova a tendência do receio/medo do novo desse grupo, ver Figura 5.

O grupo 4/4 - "Nada disso me interessa" é o menos representativo da amostra [10,50\%]. Caracterizado por indivíduos de 50 a 60 anos e/ou mais de 60 anos, viúvo(a), com renda do domicílio inferior a 1 salário mínimo e possuindo ensino fundamental como escolaridade. Esse grupo é representado por respondentes que afirmam que, realmente, nada interessa: marca conhecida, imagem/foto do produto assado e tampouco a indústria se preocupar com sabores e versões diferentes do produto tradicional. Afirmam também que discordam totalmente de que um bom pão de queijo é igual ao caseiro. $\mathrm{O}$ comportamento dos indivíduos do grupo poderia 
supor uma baixa frequência do consumo do produto tradicional e pouco interesse em obter informações ou experimentar o produto inovador. Quando questionados sobre o pão de queijo com cenoura, ficou claro que o grupo rejeita totalmente o produto, já que não lhes é nada familiar (ver Figura 6). Os resultados encontrados estão de acordo com o que foi apresentado na literatura: pessoas acima dos 50 anos declaram ser mais neofóbicas do que indivíduos de faixas etárias menores. Além disso, quanto mais baixa a renda e a escolaridade, mais alto tende a ser o nível de neofobia alimentar (TUORILA et al., 2001; BÄCKSTRÖM; PIRTTILÄ-BACKMAN; TUORILA, 2003; SCHICKENBERG et al., 2006; FLANDRIN; MONTANARI, 2009; MEISELMAN; KING; GILLETTE, 2010; SANJUÁN-LÓPEZ, PHILIPPIDIS; RESANO-EZCARAY, 2011; D'ANTUONO; BIGNAMI, 2012; FERNÁNDEZRUIZ; CLARET; CHAYA, 2013).

Quando comparados, os grupos "Pão de queijo, só se for caseiro" e "Nada disso me interessa", observa-se que a principal diferença entre eles é a representatividade da amostra, seguida da diferença de faixa etária e a da situação conjugal. Entretanto, em relação ao comportamento de rejeição de produtos alimentares não familiares, ambos os grupos tendem a rejeitar, mas com intensidades diferentes.

Assim, de modo geral, em relação aos resultados da análise de correspondência múltipla e a análise de cluster, foi possível identificar os principais fatores que determinaram as opiniões, motivações e atitudes da amostra em relação ao produto pão de queijo e o produto inovador. Entre três, dos quatro grupos de respondentes, constatou-se, por diferentes razões e graus de intensidade, tendências neofóbicas quanto ao produto alimentar inovador. Como sugestão aos agentes do setor de alimentos e interessados em lançar novidades no mercado, julga-se conveniente observar quais foram as diferenças de comportamento encontradas por este estudo entre os grupos analisados. Uma política de marketing mais eficiente poderia ser

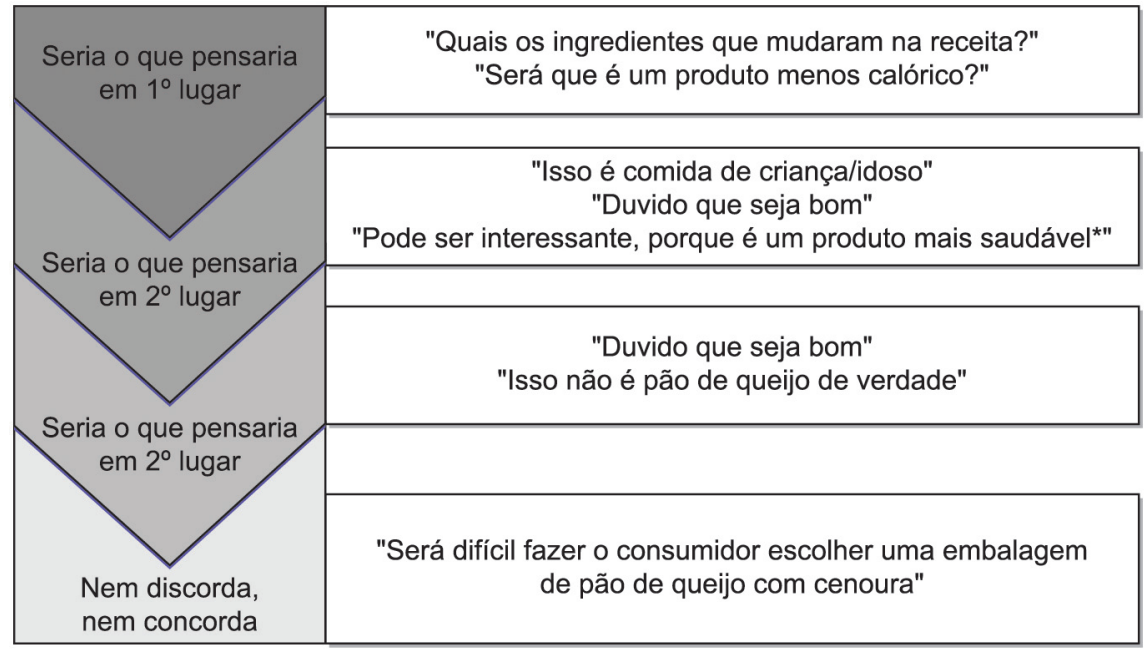

Figura 5. O que o grupo "Pão de queijo, só se for caseiro" pensa sobre um pão de queijo com cenoura. *em menor fator de impacto. Fonte: Elaborado pelas autoras.

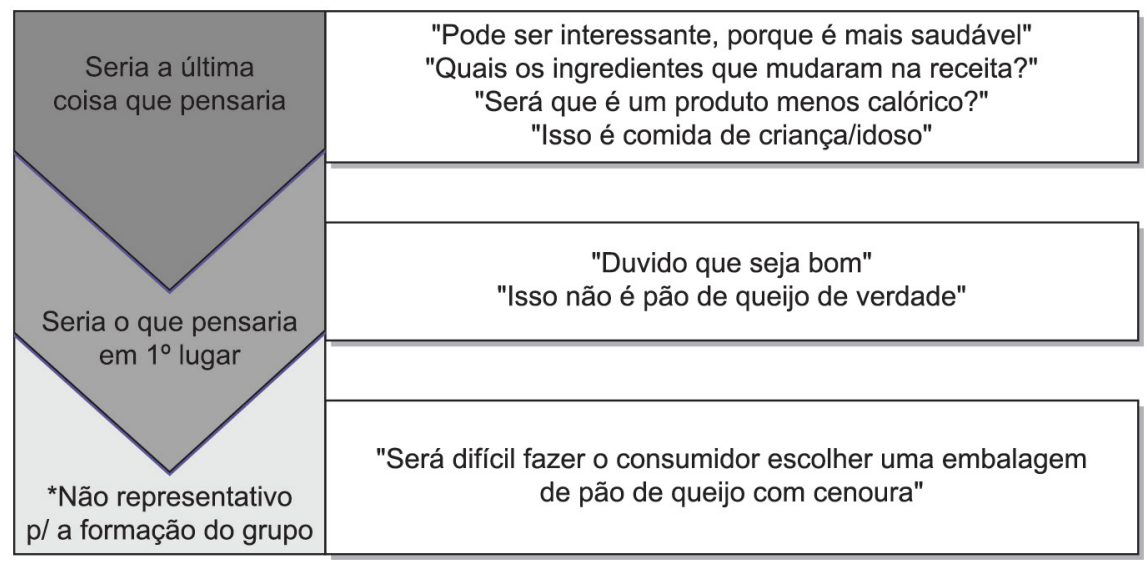

Figura 6. O que o grupo "Nada disso me interessa" pensa sobre um pão de queijo com cenoura. Fonte: Elaborado pelas autoras. 
construída a partir das constatações deste trabalho científico.

\section{Considerações finais}

Neste estudo, pretendeu-se identificar os fatores determinantes da motivação de um grupo populacional distinto quanto a um produto alimentar inovador que será lançado em breve, no mercado, o pão de queijo com cenoura. Interessou-se, principalmente, sobre o comportamento de neofobia alimentar, ou seja, sobre as razões do interesse ou da rejeição em relação ao produto alimentar não familiar. Para isso, um estudo exploratório foi realizado e 490 indivíduos, moradores da cidade de Campo Grande, MS, representaram sua amostra.

Considerando-se a urgência demandada pelos consumidores às indústrias de alimentos, sobretudo quanto à apresentação de novos produtos no mercado, poder-se-ia supor que qualquer novo produto teria ampla aceitação pelo seu caráter inovador. Contudo, os resultados deste trabalho comprovam que, de acordo com outros estudos realizados em outros países, a não familiaridade em relação a um alimento pode provocar níveis distintos de neofobia alimentar. Análises de correspondência múltipla e a de cluster comprovaram que três dos quatro grupos de respondentes encontrados apresentaram tendências de rejeição ao produto alimentar inovador/não familiar, mas em intensidades diferentes.

Apesar das limitações do estudo, ele traz importantes contribuições aos agentes dos poderes público e privado ao identificar que o produto inovador, um pão de queijo com cenoura, criado pelo SENAI/ MS, pode ser mais bem aceito entre grupos de mulheres mais abastadas e com maior nível de escolaridade da população, interessadas em novos sabores e versões desse produto. Além disso, para os indivíduos do gênero masculino, cabe à indústria respeitar a originalidade e a tradição do produto. Por fim, descobriu-se um nicho de mercado a ser pesquisado. Aquele representado por consumidores jovens que não consomem muito o produto e que têm dificuldade de categorizá-lo. Investimentos na parte visual da embalagem e informacional poderiam representar uma boa estratégia de persuasão desse nicho.

Dessa forma, todas essas informações, conseguidas pela revisão teórica e aliada aos dados empíricos, podem contribuir para as indústrias do setor. Pois o conhecimento do perfil dos consumidores e de como eles pensam sobre um produto alimentar poderia auxiliá-las na escolha de suas estratégias de segmentação de mercado, na criação de embalagem mais adequada, na promoção, na comunicação do produto nos pontos de venda, na escolha dos melhores canais de distribuição para a comercialização dos produtos alimentares e, também, na construção de marca forte para um produto alimentar inovador.

\section{Referências}

BÄCKSTRÖM, A.; PIRTTILÄ-BACKMAN, A. M.; TUORILA, H. Dimensions of novelty: a social representation approach to new foods. Appetite, v. 40, n. 3, p. 299-307, 2003. http://dx.doi.org/10.1016/ S0195-6663(03)00005-9

BLACKWELL, R. D.; MINIARD, P. W.; ENGEL, J. F. Comportamento do consumidor. São Paulo: Pioneira Thomson Learning, 2005.

BIRCH, L. L. Development of food preferences. Annual Review of Nutrition, v. 19, p. 41-62, 1999. PMid:10448516. http://dx.doi.org/10.1146/annurev. nutr.19.1.41

BUSSAB, W. O.; MORETTIN, P. A. Estatística básica. 7. ed. São Paulo: Saraiva, 2011.

CAMARENA, D.; SANJUÁN, A.; PHILIPPIDIS, G. Influence of ethnocentrism and neo-phobia on ethnic food consumption in Spain. Appetite, v. 57, n. 1, p. 121-130, 2011. PMid:21513751. http://dx.doi.org/10.1016/j. appet.2011.04.002

CRESWELL, J. W. Projeto de pesquisa: métodos qualitativo, quantitativo e misto. Tradução Luciana de Oliveira da Rocha. 2. ed. Porto Alegre: Artmed, 2007.

COOKE, L.; WARDLE, J. Age and gender differences in children's food preferences. British Journal of Nutrition, v. 93, n. 5, p. 741-746, 2005. http://dx.doi. org/10.1079/BJN20051389

DOVEY, T. M. et al. Food neophobia and 'picky/fussy' eating in children: a review. Appetite, v. 50, n. 2-3, p. 181-193, 2008. PMid:17997196. http://dx.doi.org/10.1016/j. appet.2007.09.009

D'ANTUONO, L. F.; BIGNAMI, C. Perception of typical Ukrainian foods among an Italian population. Food Quality and Preference, v. 25, n. 1, p. 1-8, 2012. http:// dx.doi.org/10.1016/j.foodqual.2011.12.003

FEDERAÇÃ̃O DAS INDÚSTRIAS DO ESTADO DE SÃO PAULO - FIESP. Brasil Food Triends 2020. São Paulo, 2010.

FISCHLER, C. L'(H)omnivore. Barcelona: Anagrama, 1995. FERNÁNDEZ-RUIZ, V.; CLARET, A.; CHAYA, C. Testing a Spanish-version of the Food Neophobia Scale. Food Quality and Preference, v. 28, n. 1, p. 222-225, 2013. http://dx.doi.org/10.1016/j.foodqual.2012.09.007

FLANDRIN, J. L.; MONTANARI, M. História da alimentação. 6. ed. São Paulo: Estação Liberdade, 2009.

FLIGHT, I.; LEPPARD, P.; COX, D. N. Food neophobia and associations with cultural diversity and socio-economic status amongst rural and urban Australian adolescents. Appetite, v. 41, n. 1, p. 51-59, 2003. http://dx.doi. org/10.1016/S0195-6663(03)00039-4

FRANK, R. A.; KLAAUW, N. V. D. The contribution of chemosensory factors to individual differences in reported food preferences, Appetite, v. 22, n. 2, p. 101-123, 1994. PMid:8037436. http://dx.doi.org/10.1006/appe.1994.1011

HURSTI, U. K.; SJÖDÉN, P. Food and general neophobia and their relationship with self-reported food choice: familial resemblance in Swedish families with children 
of ages 7-17 years. Appetite, v. 29, n. 1, p. 89-103, 1997. http://dx.doi.org/10.1006/appe.1997.0108

JOMORI, M. M.; PROENÇA, R. P. C.; CALVO, M. C. M. Determinantes de escolha alimentar. Revista de Nutrição, Campinas, v. 21, n. 1, p. 63-73, 2008.

KOIVISTO, U.; SJÖDÉN, P. Food and general neophobia in Swedish families: parent-child comparisons and relationships with serving specific foods. Appetite, v. 26, n. 2, p. 107-118, 1996. PMid:8737163. http:// dx.doi.org/10.1006/appe.1996.0009

LEBART, L.; MORINEAU, A.; PIRON, M. Statistique exploratoire miltidimensionelle. Paris: Dunod, 1995.

LUCCHESE-CHEUNG, T. Os comportamentos alimentares de brasileiros urbanos: identificação de uma tipologia de consumidores e análise das relações dos grupos com os alimentos. 2007. 237 f. Tese (Doutorado em Engenharia de Produção)-Programa de Pós-Graduação em Engenharia de Produção, Universidade Federal de São Carlos, São Carlos.

LUCCHESE-CHEUNG, T.; BATALHA, M. O.; LAMBERT, J. L. Comportamento do consumidor de alimentos: tipologia e representação da comida. Agroalimentaria, v. 18, n. 35, p. 115-129, 2012.

MALHOTRA, N. Pesquisa de marketing: foco na decisão. 3. ed. São Paulo: Pearson Prentice Hall, 2011.

MATTAR, F. N. Pesquisa de marketing. 4. ed. São Paulo: Atlas, 2007.

MACAUX, A. L. B. Eat to live or live to eat? Do parents and children agree? Public Health Nutrition, v. 4, n. 1A, p. 141-146, 2001.

MEISELMAN, H.; KING, S.; GILLETTE, M. The demographics of neophobia in a large commercial US sample. Food Quality and Preference, v. 21, n. 7, p. 893-897, 2010. http://dx.doi.org/10.1016/j. foodqual.2010.05.009

MINGOTI, S. A. Análise de dados através de métodos de estatística multivariada: uma abordagem aplicada. Belo Horizonte: UFMG, 2005. PMid:16021257.

MOONEY, K. M.; WALBOURN, L. When college students reject food: not just a matter of taste. Appetite, v. 36, n. 1, p. 41-50, 2001. PMid:11161344. http://dx.doi. org/10.1006/appe.2000.0384

NIELSEN. Tendências de saudabilidade e a luta contra a balança. 2012. Disponível em: <http://www.nielsen.com/ br/pt/insights/news/2012/tendencias-de-saudabilidadee-a-luta-contra-a-balanca.html> Acesso em: 6 set. 2013.

NIELSEN. Todo produto inovador precisa de um público encontre o seu em todos os cantos do mundo: na uncommon sense of the consumer. 2013. Disponível em: <http://www.nielsen.com/content/corporate/br/pt/ insights/reports/2013/todo-produto-inovador-precisade-um-publico-encontre-o-seu-em-todos-os-cantos. html>. Acesso em: 6 set. 2013.

NORDIN, S. et al. Gender differences in factors affecting rejection of food in healthy young Swedish adults. Appetite, v. 43, n. 3, p. 295-301, 2004. PMid:15527932. http://dx.doi.org/10.1016/j.appet.2004.07.002

PLINER, P.; HOBDEN, K. Development of a scale to measure the trait of food neophobia in humans.
Appetite, v. 19, n. 2, p. 105-120, 1992. http://dx.doi. org/10.1016/0195-6663(92)90014-W

POULAIN, J. P.; PROENÇA, R. P. C. Food social space: a tool to study food patterns. Revista de Nutrição, v. 16, n. 3, p. 245-256, 2003.

POULAIN, J. P. Sociologias da alimentação. Florianópolis: UFSC, 2004.

RITCHEY, P. et al. Validation and cross-national comparison of the food neophobia scale (FNS) using confirmatory factor analysis. Appetite, v. 40, n. 2, p. 163-173, 2003. http://dx.doi.org/10.1016/S0195-6663(02)00134-4

ROBINSON, C. H.; THOMAS, S. P. The interaction model of client health behavior as a conceptual guide in the explanation of children's health behaviors. Public Health Nutrition, v. 21, n. 1, 73-84, 2004.

SANJUÁN-LÓPEZ, A.; PHILIPPIDIS, G.; RESANOEZCARAY, H. How useful is acceptability to explain economic value? An application on the introduction of innovative saffron products into commercial markets. Food Quality and Preference, v. 22, n. 3, p. 255-263, 2011. http://dx.doi.org/10.1016/j.foodqual.2010.11.001

SCHICKENBERG, B. et al. Level of food neophobia in Dutch adults and association with familiarity with and willingness to try new healthful food products. Appetite, v. 47, n. 2, p. 257- 279, 2006.

SCHIFFMAN, L. G.; KANUK, L. L. Comportamento do consumidor. 6. ed. Rio de Janeiro: LTC, 2000.

SHETH, J.; MITTAL, B.; NEWMAN, B. Comportamento do cliente: indo além do comportamento do consumidor. São Paulo. Atlas, 2000.

SOLOMON, M. R. O comportamento do consumidor: comprando, possuindo e sendo. Porto Alegre: Bookman, 2006.

STEENKAMP, J. B. Food consumption behavior. European Advances in Consumer Research, v. 1, p. 401-409, 1993.

TUORILA, H. et al. Food neophobia among the Finns and related responses to familiar and unfamiliar foods. Food Quality and Preference, v. 12, n. 1, p. 29-37, 2001. http://dx.doi.org/10.1016/S0950-3293(00)00025-2

VENTURA, R. Mudanças no perfil do consumo no Brasil: principais tendências nos próximos 20 anos. Macroplan: Prospectiva, Estratégia e Gestão, 2010.

VERGARA, S. C. Projetos e relatórios de pesquisa em Administração. São Paulo: Atlas, 2005.

VISALBERGHI, E.; FRAGASZY, D. The behaviour of capuchin monkeys, Cebusapella, with novel food: the role of social context. Animal Behaviour, v. 49, n. 4, p. 1089-1095, 1995. http://dx.doi.org/10.1006/ anbe. 1995.0137

YAMAMOTO, M. E.; LOPES, F. A. Dize-me o que falas e te direi o que comes: aquisição da linguagem e composição da dieta em crianças. In: MOURA, M. L. S. (Org.). O bebê do século XXI e a psicologia em desenvolvimento. São Paulo: Casa do Psicólogo, 2004.

YAMAMOTO, M. E.; LOPES, F. A. A evolução do comportamento alimentar: selecionando o que comer. FAPERN, v. 1, n. 4, p. 21-24, 2006. 